\title{
Star-formation efficiency and metal enrichment of the intracluster medium in local massive clusters of galaxies
}

\author{
Y.-Y. Zhang ${ }^{1,2}$, T. F. Laganá ${ }^{3,1}$, D. Pierini ${ }^{4, \star}$, E. Puchwein ${ }^{5,6}$, P. Schneider ${ }^{1}$, and T. H. Reiprich ${ }^{1}$ \\ 1 Argelander-Institut für Astronomie, Universität Bonn, Auf dem Hügel 71, 53121 Bonn, Germany \\ e-mail: yyzhang@astro.uni-bonn.de \\ 2 National Astronomical Observatories, Chinese Academy of Sciences, Beijing 100012, PR China \\ 3 Universidade de São Paulo, Instituto de Astronomia, Geofísica e Ciências Atmosféricas, Departamento de Astronomia, \\ Rua do Matão 1226, Cidade Universitária, CEP:05508-090, São Paulo, SP, Brasil \\ ${ }^{4}$ Max-Planck-Institut für extraterrestrische Physik, Giessenbachstraße, 85748 Garching, Germany \\ 5 Heidelberger Institut für Theoretische Studien, Schloss-Wolfsbrunnenweg 35, 69118 Heidelberg, Germany \\ ${ }^{6}$ Max-Planck-Institut für Astrophysik, Karl-Schwarzschild-Straße 1, 85741 Garching, Germany
}

Received 1 March 2011 / Accepted 1 September 2011

\begin{abstract}
We have investigated the baryon-mass content in a subsample of 19 clusters of galaxies extracted from the X-ray flux-limited sample HIFLUGCS according to their positions in the sky. For these clusters, we measured total masses and characteristic radii on the basis of a rich optical spectroscopic data set, the physical properties of the intracluster medium (ICM) using XMM-Newton and ROSAT X-ray data, and total (galaxy) stellar masses utilizing the SDSS DR7 multi-band imaging. The observed (hot) gas-mass fractions are almost constant in this mass range. We confirm that the stellar mass fraction decreases as the total mass increases and shows $(20 \pm 4) \%$ scatter; in addition, we show that it decreases as the central entropy increases. The latter behavior supports a twofold interpretation, where heating from merging quenches the star-formation activity of galaxies in massive systems, and feedback from supernovae and/or radio galaxies drives a significant amount of gas to the regions beyond $r_{500}$ or, alternatively, a substantially large amount of intracluster light (ICL) is associated with galaxies in nonrelaxed systems. Furthermore, less massive clusters are confirmed to host less gas per unit total mass; however, they exhibit higher mass fractions in metals, so that their ICM is more metal-rich. This again supports the interpretation that in the potential wells of low-mass systems the star-formation efficiency of galaxies was high or, alternatively, some gas is missing from the hot phase of the ICM. The former hypothesis is preferred as the main driver of the mass-dependent metal enrichment since the total mass-to-optical luminosity ratio increases as the total mass increases.
\end{abstract}

Key words. X-rays: galaxies: clusters - cosmology: observations - methods: data analysis - galaxies: clusters: general - surveys galaxies: stellar content

\section{Introduction}

Galaxy clusters are the largest gravitationally bound structures in the Universe, and they have become an important cosmological probe to constrain dark energy (e.g., Vikhlinin et al. 2009). Calibrating the baryon content of galaxy clusters is thus a key input for understanding the structure-formation history, as well as systematic uncertainties in constraining cosmology with galaxy clusters as standard candles.

The hot intracluster medium (ICM; e.g., Sarazin \& Bahcall 1977) and the stars bound to member galaxies have been considered to be the main contributors to the baryon content in clusters. The baryon-mass fraction of a galaxy cluster was thus defined as the sum of the gas-mass fraction $\left(f_{\text {gas }}:=M_{\text {gas }} / M_{\text {tot }}\right.$, the gas-tototal mass ratio) and the stellar mass fraction $\left(f_{*}:=M_{*} / M_{\text {tot }}\right.$, the stellar-to-total mass ratio in which the stars in galaxies are considered). We can use X-ray data alone to measure the gasmass fraction (e.g., Ettori et al. 2002; Zhang et al. 2006). There are two well-established methods of measuring the total stellar mass in a galaxy cluster. One is to measure the stellar mass for the total galaxy population of a cluster from the total luminosity based on the galaxy luminosity function (GLF) and

\footnotetext{
* Guest astronomer at the MPE.
}

a stellar mass-to-optical light ratio that depends on the mass of a cluster (e.g., David et al. 1990; Roussel et al. 2000; Lin et al. 2003). The other is to measure the stellar masses of the individual member galaxies and to construct the stellar mass function in order to sum these stellar masses (e.g., see Sect. 3 in Giodini et al. 2009). The contribution to the total stellar mass budget associated with intracluster light (ICL; e.g., Zibetti et al. 2005) could be significant especially on the mass scale of galaxy groups if the ICL makes more than $10-20 \%$ of the total light (e.g., Gonzalez et al. 2007). The discrepancies between measurements of the baryon-mass fraction of galaxy clusters and groups (e.g., Lin et al. 2003; Gonzalez et al. 2007; Krick \& Bernstein 2007; Laganá et al. 2008, 2011; Giodini et al. 2009; Andreon 2010; Dai et al. 2010) and the measurement from the Wilkinson Microwave Anisotropy Probe (WMAP) 5-year data (Dunkley et al. 2009) allow for an ICL-to-total stellar mass ratio in a wide range up to $\sim 50 \%$ with a controversial mass dependence. Most observational studies do not support dominant contributions from the ICL (e.g., Zibetti et al. 2005; see Arnaboldi \& Gerhard 2010, for a recent review). Furthermore, theoretical studies reach controversial conclusions on the origin and mass dependence of the ICL fraction (e.g., Murante et al. 2004; Dolag et al. 2010, and references therein). 
Table 1. Properties of the 19 galaxy clusters.

\begin{tabular}{|c|c|c|c|c|c|c|c|c|}
\hline \multirow[t]{2}{*}{ Name } & \multicolumn{2}{|c|}{ X-ray center $(\mathrm{J} 2000)$} & \multirow[t]{2}{*}{ Redshift } & \multirow{2}{*}{$\begin{array}{c}M_{500} \\
10^{14} M_{\odot}\end{array}$} & \multirow{2}{*}{$\begin{array}{c}M_{500, M-M_{\mathrm{gas}}} \\
10^{14} M_{\odot}\end{array}$} & \multirow{2}{*}{$\begin{array}{c}M_{\text {gas, } 500} \\
10^{13} M_{\odot}\end{array}$} & \multirow{2}{*}{$\begin{array}{c}M_{*, 500} \\
10^{12} M_{\odot}\end{array}$} & \multirow{2}{*}{$\begin{array}{l}\text { Undisturbed } \\
\text { /Cool core }\end{array}$} \\
\hline & RA & Dec & & & & & & \\
\hline $\mathrm{A} 0085$ & $00: 41: 50.306$ & $-09: 18: 11.11$ & 0.0556 & $6.37 \pm 1.00$ & $5.68 \pm 0.37$ & $8.13 \pm 0.38$ & $2.71 \pm 1.83$ & $\mathrm{Y} / \mathrm{S}$ \\
\hline A0400 & $02: 57: 41.349$ & $+06: 01: 36.93$ & 0.0240 & $1.83 \pm 0.39$ & $1.07 \pm 0.07$ & $1.36 \pm 0.05$ & $1.58 \pm 1.07$ & $\mathrm{~N} / \mathrm{N}$ \\
\hline IIIZw54 & $03: 41: 18.729$ & $+15: 24: 13.91$ & 0.0311 & $1.91 \pm 0.58$ & $1.18 \pm 0.08$ & $1.45 \pm 0.26$ & $2.79 \pm 1.56$ & $\mathrm{Y} / \mathrm{W}$ \\
\hline A1367 & $11: 44: 44.501$ & $+19: 43: 55.82$ & 0.0216 & $1.76 \pm 0.27$ & $2.11 \pm 0.14$ & $2.07 \pm 0.07$ & $1.72 \pm 0.30$ & $\mathrm{~N} / \mathrm{N}$ \\
\hline MKW4 & 12:04:27.660 & $+01: 53: 41.50$ & 0.0200 & $0.50 \pm 0.14$ & $0.58 \pm 0.04$ & $0.47 \pm 0.02$ & $0.63 \pm 0.18$ & $\mathrm{Y} / \mathrm{S}$ \\
\hline ZwCl1215 & $12: 17: 40.637$ & $+03: 39: 29.66$ & 0.0750 & $4.93 \pm 0.98$ & $4.34 \pm 0.28$ & $6.10 \pm 0.29$ & $3.06 \pm 2.30$ & $\mathrm{Y} / \mathrm{N}$ \\
\hline A 1650 & $12: 58: 41.885$ & $-01: 45: 32.91$ & 0.0845 & $3.44 \pm 0.66$ & $4.28 \pm 0.27$ & $5.09 \pm 0.73$ & $3.09 \pm 1.43$ & $\mathrm{Y} / \mathrm{W}$ \\
\hline Coma & $12: 59: 45.341$ & $+27: 57: 05.63$ & 0.0232 & $6.55 \pm 0.79$ & $6.21 \pm 0.40$ & $8.42 \pm 0.63$ & $2.23 \pm 1.12$ & $\mathrm{~N} / \mathrm{N}$ \\
\hline A1795 & $13: 48: 52.790$ & $+26: 35: 34.36$ & 0.0616 & $3.41 \pm 0.63$ & $4.46 \pm 0.29$ & $5.11 \pm 0.14$ & $2.44 \pm 0.81$ & $\mathrm{Y} / \mathrm{S}$ \\
\hline MKW8 & $14: 40: 42.150$ & $+03: 28: 17.87$ & 0.0270 & $0.62 \pm 0.12$ & $1.10 \pm 0.07$ & $0.80 \pm 0.12$ & $0.52 \pm 0.39$ & $\mathrm{~N} / \mathrm{N}$ \\
\hline A2029 & $15: 10: 55.990$ & $+05: 44: 33.64$ & 0.0767 & $14.70 \pm 2.61$ & $6.82 \pm 0.44$ & $13.35 \pm 0.53$ & $4.49 \pm 0.80$ & $\mathrm{Y} / \mathrm{S}$ \\
\hline A2052 & $15: 16: 44.411$ & $+07: 01: 12.57$ & 0.0348 & $1.39 \pm 0.28$ & $2.03 \pm 0.13$ & $1.86 \pm 0.10$ & $2.84 \pm 0.33$ & $\mathrm{Y} / \mathrm{S}$ \\
\hline MKW3S & $15: 21: 50.277$ & $+07: 42: 11.77$ & 0.0450 & $1.45 \pm 0.34$ & $2.29 \pm 0.15$ & $2.13 \pm 0.09$ & $1.30 \pm 1.76$ & $\mathrm{Y} / \mathrm{S}$ \\
\hline A2065 & $15: 22: 29.082$ & $+27: 43: 14.39$ & 0.0721 & $11.18 \pm 1.78$ & $3.35 \pm 0.22$ & $7.66 \pm 1.44$ & $3.04 \pm 1.53$ & $\mathrm{~N} / \mathrm{W}$ \\
\hline A 2142 & $15: 58: 19.776$ & $+27: 14: 00.96$ & 0.0899 & $7.36 \pm 1.25$ & $10.26 \pm 0.66$ & $13.76 \pm 0.73$ & $4.04 \pm 2.22$ & $\mathrm{Y} / \mathrm{W}$ \\
\hline A2147 & $16: 02: 16.305$ & $+15: 58: 18.46$ & 0.0351 & $4.44 \pm 0.67$ & $3.63 \pm 0.23$ & $5.04 \pm 0.53$ & $2.37 \pm 1.76$ & $\mathrm{~N} / \mathrm{N}$ \\
\hline A2199 & $16: 28: 37.126$ & $+39: 32: 53.29$ & 0.0302 & $2.69 \pm 0.42$ & $2.64 \pm 0.17$ & $2.97 \pm 0.30$ & $2.26 \pm 0.06$ & $\mathrm{Y} / \mathrm{S}$ \\
\hline A 2255 & $17: 12: 54.538$ & $+64: 03: 51.46$ & 0.0800 & $7.13 \pm 1.38$ & $4.08 \pm 0.26$ & $7.11 \pm 0.33$ & $2.99 \pm 1.79$ & $\mathrm{~N} / \mathrm{N}$ \\
\hline A2589 & $23: 23: 56.772$ & $+16: 46: 33.19$ & 0.0416 & $3.03 \pm 0.75$ & $1.88 \pm 0.12$ & $2.54 \pm 0.17$ & $1.77 \pm 2.04$ & $\mathrm{Y} / \mathrm{W}$ \\
\hline
\end{tabular}

Notes. The cluster mass, $M_{500, M-M_{\text {gas }}}$, is derived from the $M_{500}-M_{\text {gas }, 500}$ relation as tabulated in Laganá et al. (2011), and only used for comparison with the cluster mass, $M_{500}$, derived from the "harmonic" velocity dispersion. "S", "W", and "N" denote strong cool-core, weak cool-core, and noncool-core clusters (see Hudson et al. 2010).

Understanding the baryon-mass fraction and its mass dependence will be a milestone to understand astrophysics in galaxy clusters, e.g., the origin of the ICL (e.g., Pierini et al. 2008), starformation history (e.g., Fritz et al. 2010), and metal-enrichment history (e.g., Kapferer et al. 2009). This also helps to control systematic uncertainties in high-precision cluster cosmology.

The HIFLUGCS (Reiprich \& Böhringer 2002) provides an X-ray flux-limited sample of 64 nearby $(z<0.1)$ clusters selected from the ROSAT All-Sky Survey (RASS). Nineteen clusters in the HIFLUGCS have been imaged beyond $9 r_{500}{ }^{1}$ in the Sloan Digital Sky Survey (SDSS, DR7, Abazajian et al. 2009). Since these 19 clusters were selected from the HIFLUGCS based on their sky positions, this subsample is unbiased with respect to the HIFLUGCS. Our sample has therefore inherited the nature of the flux selection.

In this paper, we analyze XMM-Newton and ROSAT X-ray data, as well as optical data for this sample of those 19 clusters, and investigate the baryon content in clusters and its mass dependence. This study, similar to Andreon (2010), has the advantage over many previous studies that the cluster total masses and reference radii are derived from the velocity dispersion based on optical spectroscopic data (see Zhang et al. 2011, and references therein) that are independent of both the X-ray data used to determine the gas masses and the optical imaging data used to measure the stellar masses. It enables a relatively objective investigation of the intrinsic scatter in the gas-mass, stellar-mass and baryon-mass fractions including contributions from the intrinsic scatter of mass estimates.

We organize the paper as follows. In Sect. 2, we describe the sample and data analysis. We present our results in Sect. 3, as well as discussions in Sect. 4, and summarize our findings

\footnotetext{
1 The cluster radius, $r_{\Delta}$, is the radius within which the mass density is $\Delta$ times the critical density, $\rho_{\mathrm{c}}(z)=E^{2}(z) 3 H_{0}^{2} /(8 \pi G)$, where $E^{2}(z)=\Omega_{\Lambda}+\Omega_{\mathrm{m}}(1+z)^{3}+\left(1-\Omega_{\Lambda}-\Omega_{\mathrm{m}}\right)(1+z)^{2}$. In this work, we measured two cluster radii, $r_{500}$ and $r_{200}$. As shown in Laganá et al. (2011), the coverage of $9 r_{500}$ is required to perform a robust background subtraction to construct the GLF of the cluster.
}

in Sect. 5. We assume $\Omega_{\mathrm{m}}=0.3, \Omega_{\Lambda}=0.7$, and $H_{0}=$ $70 \mathrm{~km} \mathrm{~s}^{-1} \mathrm{Mpc}^{-1}$. Throughout this paper we apply the BCES regression fitting method taking measurement errors into account in both variables and their covariance (Akritas \& Bershady 1996). Confidence intervals correspond to the $68 \%$ confidence level.

\section{Sample and data}

\subsection{Sample}

The HIFLUGCS is an X-ray flux-limited sample of 64 nearby $(z<0.1)$ clusters selected from the RASS. Nineteen clusters (Table 1) in the HIFLUGCS have available XMM-Newton and SDSS DR7 data (Abazajian et al. 2009).

\subsection{Cluster total mass and radius}

Recent observations suggest deviations from hydrostatic equilibrium in galaxy clusters (e.g., Zhang et al. 2008, 2010; Mahdavi et al. 2008). We therefore avoid using the X-ray hydrostatic mass (e.g., Zhang et al. 2009) or caustic mass (e.g., Rines \& Diaferio 2006) as the cluster mass. Furthermore, to guarantee a fair study, we choose to obtain the total mass from a quantity that is not linked to either quantities that we study, i.e., stellar mass and gas mass.

We calculate the cluster mass from the velocity dispersion using the optical spectroscopic data listed in Table 1 in Zhang et al. (2011, also see references therein), which are independent of both the X-ray data used to derive the ICM properties and the optical SDSS imaging data used to derive the stellar masses.

Taking the "harmonic" velocity dispersion $\left(\sigma_{\mathrm{a}, \mathrm{p}}\right)$ measured within an aperture of 1.2 Abell radii $(a=2.57 \mathrm{Mpc})$ from Zhang et al. (2011), we follow the method described in Sect. 3 in Biviano et al. (2006) of computing the cluster mass $M_{\sigma}$. We first determine an initial estimate of the mass from $\sqrt{3} \sigma_{\mathrm{a}, \mathrm{p}}$ through Eq. (2) in Biviano et al. (2006). An estimate of the radius $\tilde{r_{\mathrm{v}}}$ is 
derived through steps 7 and 9 in Biviano et al. (2006). Replacing the true quantities $r_{\mathrm{v}}$ and $\sigma_{\mathrm{a}}$ with their estimates $\tilde{r_{\mathrm{v}}}$ and $\sqrt{3} \sigma_{\mathrm{a}, \mathrm{p}}$ in Fig. 4 in Biviano et al. (2006), we obtain an estimate of $\sigma_{\mathrm{v}}$. The cluster mass $M_{\sigma}$ is given by Eq. (2) in Biviano et al. (2006) and is accurate to $\approx 10 \%$. The mass error is derived by combining in quadrature the error in the velocity dispersion converted in mass, additional velocity dispersion error introduced by the uncertainty in Fig. 4 in Biviano et al. (2006), and the 10\% accuracy in $M_{\sigma}$. Typical mass errors are $\sim 20 \%$ (Table 1 ). To compute $r_{500}$ from $M_{200}$, we assume an NFW (Navarro et al. 1997) model with the concentration parameter given in step 7 in Biviano et al. (2006).

As shown in Fig. 3 in Zhang et al. (2011), the HIFLUGCS itself is biased toward X-ray luminous clusters at the low-mass end and contains an increasing fraction of mergers toward the high-mass end. This leads to a large fraction of X-ray luminous clusters in the low-mass regime and a dominant fraction of mergers in the high-mass regime for the sample of 19 clusters. The velocity dispersion estimates can be biased high by substructures, which leads to overestimating the dynamical masses for mergers (e.g., Biviano et al. 2006). On the other hand, the cluster masses, derived from our best fit of the X-ray hydrostatic mass versus gas mass relation of the clusters and groups in Vikhlinin et al. (2006), Arnaud et al. (2007), Pratt et al. (2009), and Sun et al. (2009), are biased high for the X-ray luminous clusters in the low-mass regime. Consequently, we observe that, at the lowmass/high-mass end, the total masses derived from the velocity dispersion appear low/high compared to the total masses derived from the gas masses as shown in Fig. 1. The two most outliers are A2029 and A2065, which have the highest masses derived from the "harmonic" velocity dispersion. Therefore, the full sample of the 19 clusters and a sample of 17 clusters excluding A2029 and A2065 will be investigated respectively, in Sect. 3 .

We derived all quantities consistently within the cluster radius, $r_{500}$, unless explicitly stated otherwise.

\subsection{X-ray data}

Details on the joint X-ray XMM-Newton and ROSAT data analysis and properties of the XMM-Newton observations can be found in Zhang et al. (2009, 2011). The procedures for cleaning the data are described in Sect. 2.2 and for detecting and subtracting point-like sources in Sect. 2.3 in Zhang et al. (2009). The background treatment can be found in Sect. 2.4 of Zhang et al. (2009). Significant substructure features clearly detected in the image are excised before we perform the spectral and surface brightness analysis.

The truncation radii of the XMM-Newton observed surface brightness profiles, within which the signal-to-noise ratio $(S / N)$ is greater than 3 , are rather small $\left(<r_{500}\right)$. The ROSAT observed surface brightness profiles cover radii well beyond $r_{500}$ with $S / N \geq 3$, although with sparse data points to resolve the cluster core. As noted in Sect. 2.2 in Zhang et al. (2011), we directly convert the ROSAT surface brightness profile to the XMM-Newton count rate using the best-fit spectral model obtained from the XMM-Newton data. We then combine the $X M M-N e w t o n$ surface brightness profile within the truncation radius, where the $X M M$-Newton $S / N$ is $\sim 3$, with the ROSAT converted surface brightness profile beyond the truncation radius for further analysis.

The soft band (0.7-2 keV) X-ray surface brightness profile model $S_{\mathrm{X}}(R)$, in which $R$ is the projected radius, is linked to the ICM radial electron number density profile $n_{\mathrm{e}}(r)$ and emissivity

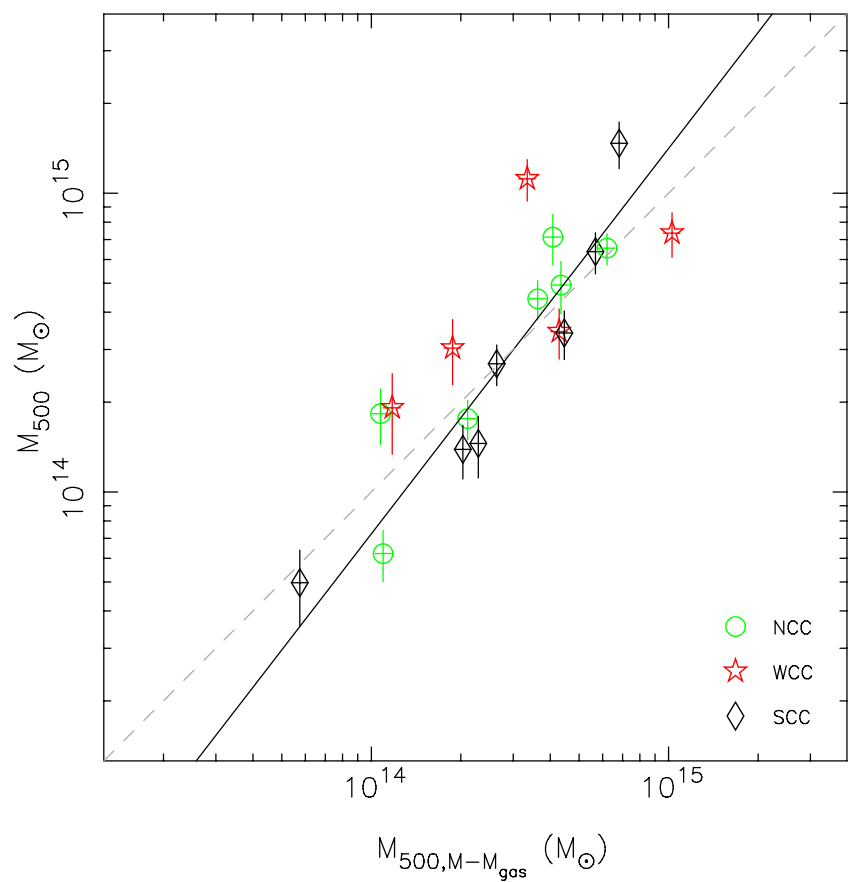

Fig. 1. Cluster mass derived from the velocity dispersion compared to the cluster mass derived from the gas mass using the mass vs. gas mass relation. The solid line shows the best fit. The dashed line denotes the 1:1 ratio. The green circles, red stars, and black diamonds stand for noncool-core, weak cool-core and strong cool-core clusters, respectively. The two most outliers are A2029 and A2065, which have the highest masses derived from the harmonic velocity dispersion.

function as an integral performed along the line of sight convolved with the $X M M$-Newton point-spread function (PSF) as a function of the detector coordinates $(X, Y)$,

$S_{\mathrm{X}}(R) \propto \iiint \operatorname{PSF}(X, Y, R) n_{\mathrm{e}}^{2}\left(\sqrt{l^{2}+R^{2}}\right) \mathrm{d} \ell \mathrm{d} X \mathrm{~d} Y$.

The gas mass distribution is derived by integrating the gas density distribution. Gas masses are measured with respect to $r_{500}$ derived in Sect. 2.2. Errors are derived by combining in quadrature measurement errors of gas masses at $r_{500}$ and errors due to the variation of $r_{500}$ within its $1 \sigma$ interval. Typical gas mass errors are within $10 \%$ (see Table 1).

The spectral analysis through which we obtain the radial temperature distribution $T(r)$ is documented in Sect. 3 in Zhang et al. (2009). The radial entropy distribution is computed as $S(r)=T(r) n_{\mathrm{e}}^{-2 / 3}(r)$. The XMM-Newton data enable measuring the radial temperature distribution beyond $0.2 r_{500}$. The central entropies are thus measured at $0.1 r_{500}$ and $0.2 r_{500}$.

\subsection{Optical imaging data}

The SDSS ${ }^{2}$ DR7 has the advantage over previous SDSS data releases of using more reliable sky background subtraction (Abazajian et al. 2009). We used the magnitudes in the "dered" table in the "PHOTO" catalog. Hereafter we briefly summarize the procedure on constructing the GLF of the galaxy cluster (see Laganá et al. 2008, 2011, for details) as follows.

2 The $K$-band luminosity from the Two Micron All Sky Survey (2MASS) observations can also be used to measure the stellar mass (Lin et al. 2003). Compared to the 2MASS observations, the SDSS data allow us to make a more reliable selection of cluster member galaxies, which greatly reduces fore- and background contaminations. 
Table 2. Slopes and characteristic magnitudes of the double Schechter function fit to the GLF, gas-mass fraction, and stellar mass fraction.

\begin{tabular}{lcccccc}
\hline \hline Name & $\alpha_{1}$ & $M_{*, 1}$ & $\alpha_{2}$ & $M_{*, 2}$ & $f_{\text {gas }, 500}$ & $f_{*, 500}$ \\
\hline A0085 & $-1.484 \pm 0.045$ & $-21.87 \pm 0.71$ & $-1.945 \pm 0.072$ & $-20.30 \pm 0.05$ & $0.128 \pm 0.010$ & $0.0115 \pm 0.0012$ \\
A0400 & $-1.194 \pm 0.057$ & $-22.24 \pm 0.11$ & $-1.821 \pm 0.027$ & $-18.94 \pm 0.06$ & $0.075 \pm 0.008$ & $0.0240 \pm 0.0039$ \\
IIIZw54 & $-1.289 \pm 0.120$ & $-22.00 \pm 0.70$ & $-1.651 \pm 0.028$ & $-18.42 \pm 0.01$ & $0.076 \pm 0.013$ & $0.0239 \pm 0.0039$ \\
A1367 & $-1.184 \pm 0.029$ & $-22.72 \pm 0.81$ & $-1.922 \pm 0.062$ & $-18.98 \pm 0.04$ & $0.118 \pm 0.009$ & $0.0247 \pm 0.0028$ \\
MKW4 & $-1.666 \pm 0.073$ & $-20.62 \pm 0.35$ & $-1.802 \pm 0.024$ & $-19.65 \pm 0.10$ & $0.094 \pm 0.013$ & $0.0233 \pm 0.0040$ \\
ZwC11215 & $-1.698 \pm 0.013$ & $-22.02 \pm 0.11$ & - & - & $0.124 \pm 0.013$ & $0.0143 \pm 0.0017$ \\
A1650 & $-1.271 \pm 0.051$ & $-23.10 \pm 0.60$ & - & - & $0.148 \pm 0.018$ & $0.0217 \pm 0.0026$ \\
Coma & $-1.303 \pm 0.025$ & $-22.34 \pm 0.36$ & $-1.935 \pm 0.019$ & $-19.76 \pm 0.08$ & $0.129 \pm 0.009$ & $0.0201 \pm 0.0018$ \\
A1795 & $-1.327 \pm 0.006$ & $-21.48 \pm 0.61$ & $-1.847 \pm 0.032$ & $-20.17 \pm 0.02$ & $0.150 \pm 0.014$ & $0.0182 \pm 0.0022$ \\
MKW8 & $-1.145 \pm 0.074$ & $-20.55 \pm 0.45$ & $-1.959 \pm 0.028$ & $-19.32 \pm 0.02$ & $0.128 \pm 0.016$ & $0.0259 \pm 0.0031$ \\
A2029 & $-1.227 \pm 0.010$ & $-21.83 \pm 0.86$ & - & - & $0.091 \pm 0.008$ & $0.0065 \pm 0.0007$ \\
A2052 & $-1.313 \pm 0.005$ & $-21.74 \pm 0.81$ & $-1.964 \pm 0.014$ & $-19.68 \pm 0.01$ & $0.134 \pm 0.014$ & $0.0254 \pm 0.0030$ \\
MKW3S & $-1.422 \pm 0.033$ & $-22.62 \pm 0.49$ & $-1.822 \pm 0.036$ & $-19.81 \pm 0.03$ & $0.147 \pm 0.017$ & $0.0268 \pm 0.0035$ \\
A2065 & $-1.061 \pm 0.029$ & $-22.01 \pm 0.26$ & $-1.643 \pm 0.015$ & $-20.46 \pm 0.01$ & $0.069 \pm 0.008$ & $0.0065 \pm 0.0006$ \\
A2142 & $-1.271 \pm 0.014$ & $-21.87 \pm 0.10$ & $-1.495 \pm 0.094$ & $-20.38 \pm 0.01$ & $0.187 \pm 0.017$ & $0.0114 \pm 0.0011$ \\
A2147 & $-1.304 \pm 0.009$ & $-21.52 \pm 0.12$ & $-1.879 \pm 0.015$ & $-19.91 \pm 0.01$ & $0.114 \pm 0.010$ & $0.0154 \pm 0.0015$ \\
A2199 & $-1.154 \pm 0.031$ & $-21.44 \pm 0.34$ & $-2.024 \pm 0.014$ & $-19.65 \pm 0.01$ & $0.110 \pm 0.010$ & $0.0177 \pm 0.0017$ \\
A2255 & $-1.419 \pm 0.024$ & $-23.02 \pm 0.98$ & - & - & $0.100 \pm 0.010$ & $0.0095 \pm 0.0011$ \\
A2589 & $-1.085 \pm 0.013$ & $-21.71 \pm 0.17$ & $-1.838 \pm 0.021$ & $-19.89 \pm 0.01$ & $0.084 \pm 0.011$ & $0.0169 \pm 0.0023$ \\
\hline
\end{tabular}

A color-magnitude diagram is constructed for the galaxies within a projected radius of $r_{500}$. The galaxies with $0.7<$ $(g-i)<1.6$ and $i<18$ in the $g-i$ vs. $i$ diagram $^{3}$ are bright and red galaxies, and are used to make a preliminary fit of the red sequence in the color-magnitude diagram with a linear relation. Iteratively, the red sequence is fitted using those galaxies in the full magnitude range within $3 \sigma$ redward and blueward of the previous red-sequence fit until the fit converges. The red galaxies are defined as those within 0.3 mag redward and blueward in $g-i$ of the best-fit red sequence, and blue galaxies as those bluer than the lower limit for the red galaxy selection.

The fore- and background galaxies are subtracted statistically (e.g., Zwicky 1957; Oemler 1973). The $i$-band GLF is constructed for the selected red and blue galaxies with a bin size of 0.5 mag. The GLF of the background galaxies is derived using those galaxies within an annular region located beyond $8 r_{500}$ from the X-ray flux-weighted cluster center. A power-law model is fitted to the GLF of the background galaxies and subtracted from the overall GLF. A fair background subtraction should not introduce major uncertainties since the GLF is sampled well enough at the bright end, and it does not abruptly rise at the faint end. Large-scale structures (LSSs) may introduce some uncertainties into this kind of background subtraction. Recent tests (e.g., Paolillo et al. 2001; Gonzalez et al. 2007; De Filippis et al. 2011) show that the difference in the GLF is within $10 \%$ compared to the case using the background level derived from the SDSS GLF (e.g., Blanton et al. 2003), integrating over redshift and applying mean evolutionary and $K$-corrections (e.g., Bruzual \& Charlot 2003) with the Padova isochrones (Bertelli et al. 1994). Corrections due to poor or absent sampling of the faint end of the GLF should only be a few per cent (see Andreon 2010).

There are two obvious components in the GLF for most clusters. A double Schechter (1976) function is thus used to fit the GLF of each cluster, in which the covariance between the power-law index at the faint end $(\alpha)$ and the characteristic magnitude $\left(M^{*}\right)$ is taken into account (see Table 2). The $K$-correction for early-type galaxies and the redshift evolution

\footnotetext{
3 This magnitude cut best suits the majority of the 19 clusters. For four clusters (IIIZw54, A1367, MKW4, and A400) we apply $i<16$, which better defines the preliminary slope of the red sequence.
}

correction given in Poggianti (1997) are adopted to correct the characteristic magnitudes. The total $i$-band luminosity is derived by integrating the double Schechter function for magnitudes brighter than -14 .

The mass-to-light ratios for ellipticals and spirals, respectively, following Kauffmann et al. (2003) assuming the Salpeter (1955) initial mass function (IMF) are adopted to compute the stellar mass from the optical luminosity. The stellar mass estimate is tied to the choice of IMF. Changing the IMF will scale the stellar mass estimate by a fixed factor, e.g., from a Kroupa (2001) IMF to a Salpeter (1955) IMF with a cut-off at $0.1 M_{\odot}$ results in a factor of 2 increase in the stellar mass (e.g., Kauffmann et al. 2003).

Any systematic offset in the spectrophotometric calibration will change the stellar mass estimate (e.g., Kauffmann et al. 2003). Fritz et al. (2010) have carried out a thorough comparison of stellar mass estimates for a large sample of more than 50 nearby clusters $(z<0.07)$ computed from spectroscopic and photometric data. They found that the stellar masses computed from the fiber-aperture magnitude using SDSS DR7 photometric data are lower by $\sim 0.15$ dex than the values computed from the spectroscopic data within the same aperture (also see Andreon 2010). Our sample is in similar redshift and mass ranges as their sample, and we also calculated the total stellar mass using the SDSS DR7 data. Therefore, this bias is corrected in our total stellar mass estimates.

\section{Results}

Figure 2 shows the gas-mass, stellar mass, and baryon-mass fractions as a function of the total mass. We also compared our observational sample to some existing observational samples (e.g., Lin et al. 2003; Gonzalez et al. 2007; Giodini et al. 2009; Pratt et al. 2009; Sun et al. 2009; Andreon 2010; Dai et al. 2010), as well as simulated samples (e.g., Puchwein et al. 2008, 2010; Fabjan et al. 2010).

\subsection{Gas-mass fraction}

The gas-mass fractions of the 19 clusters appear flat in the given narrow mass range with $(26 \pm 4) \%$ intrinsic scatter. Excluding 
Y.-Y. Zhang et al.: Star-formation efficiency and metal enrichment of the ICM in local massive clusters of galaxies

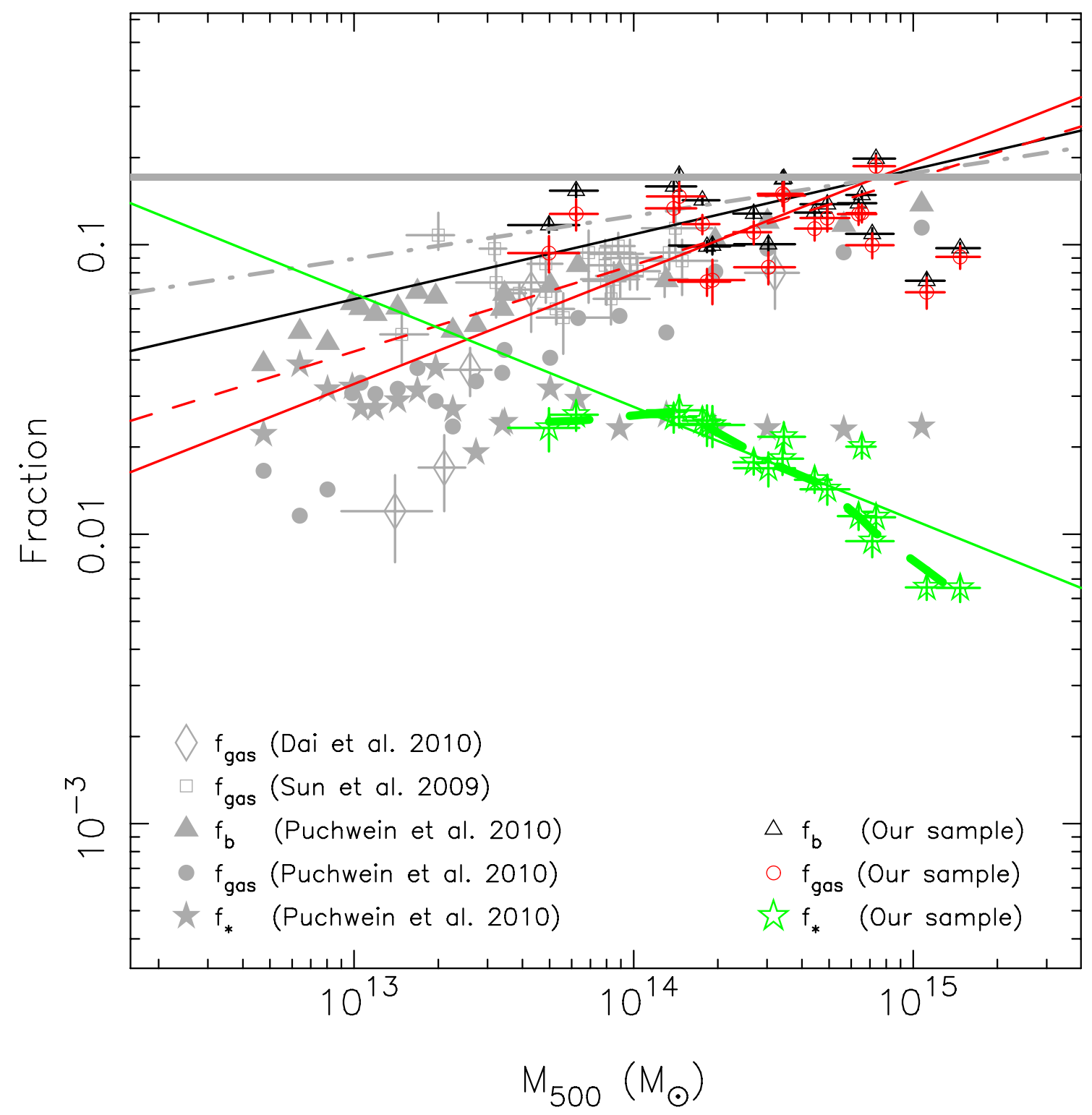

Fig. 2. Gas-mass fraction (red, open circles), stellar mass fraction (green, open stars), and baryon-mass fraction (black, open triangles) as a function of the total mass and the best fits excluding A2065 and A2029 in the same colors with solid line. The stellar mass fraction in our observational sample decreases with increasing cluster mass with $(20 \pm 4) \%$ intrinsic scatter for the 19 clusters. For comparison, we also show the gas-mass fraction (gray, solid circles), stellar mass fraction in galaxies (gray, solid stars), and the sum of these two fractions (gray, solid triangles) for the simulated sample of 21 clusters with AGN feedback in Puchwein et al. (2010), as well as the gas mass fractions of the X-ray selected groups in Sun et al. (2009; gray, open boxes) and near-infrared selected groups in Dai et al. (2010; gray, open diamonds). The best fit of the baryon-mass fraction as a function of the total mass of the observational sample in Lin et al. (2003) is shown in gray with dot-dashed line. The gray band shows the $1 \sigma$ measurement from the WMAP 5-year result (Dunkley et al. 2009). A2142 displays a baryon-mass fraction of $0.198 \pm 0.008$, which exceeds the WMAP result with a $3 \sigma$ significance. The best fit of the gas-mass fractions combining our clusters and the clusters in Sun et al. (2009) is shown in red with dashed line with $(26 \pm 8) \%$ intrinsic scatter.

the two most prominent outliers, A2029 and A2065, shown in Sect. 2.2, the gas-mass fraction increases with the total mass as $f_{\text {gas }, 500}=10^{-(1.10 \pm 0.16)}\left(M_{500} /\left[10^{14} M_{\odot}\right]\right)^{0.38 \pm 0.36}$ with $(23 \pm 17) \%$ intrinsic scatter. To constrain the slope precisely, we combine our 19 clusters and the groups in Sun et al. (2009), which show an increasing gas-mass fraction as a function of the total mass, i.e., $f_{\text {gas }, 500}=10^{-(1.07 \pm 0.02)}\left(M_{500} /\left[10^{14} M_{\odot}\right]\right)^{0.30 \pm 0.07}$ with $(26 \pm 8) \%$ intrinsic scatter. This shows the importance of having a wide mass range to calibrate the mass dependence of the gas-mass fraction.

The normalization for our sample is comparable to what is obtained for the X-ray selected cluster sample in
Pratt et al. (2009) and X-ray selected group sample in Sun et al. (2009), and is higher than the 2MASS selected stacked groups in Dai et al. (2010) with $>3 \sigma$ significance. This indicates that to some extent X-ray selected groups may be biased toward systems with high gas-mass fractions, whereas near-infrared (or optically) selected groups are biased toward systems with low gasmass fractions. (The extreme systems are probably the so-called X-ray under-luminous systems, e.g., Dietrich et al. 2009.)

The gas-mass fractions of our observational sample agree well with the predictions of the simulated sample, in particular, in the high-mass regime, i.e., $\geq 10^{14} M_{\odot}$. It is interesting that the slope of the mock sample with AGN feedback is between 
Table 3. Comparison of the best fits of the gas-mass fraction, stellar mass fraction, and baryon-mass fraction as a function of the cluster total mass between different samples.

\begin{tabular}{lcc}
\hline \hline Sample & Best fit & Intrinsic scatter \\
\hline 19 clusters & $f_{\text {gas }, 500}=10^{-(0.93 \pm 0.32)}\left(M_{500} /\left[10^{14} M_{\odot}\right]\right)^{-(0.03 \pm 0.64)}$ & $(26 \pm 4) \%$ \\
19 clusters-A2029-A2065 & $f_{\text {gas } 500}=10^{-(1.10 \pm 0.16)}\left(M_{500} /\left[10^{14} M_{\odot}\right]\right)^{0.38 \pm 0.36}$ & $(23 \pm 17) \%$ \\
19 clusters and Sun+09 & $f_{\text {gas,500 }}=10^{-(1.07 \pm 0.02)}\left(M_{500} /\left[10^{14} M_{\odot}\right]\right)^{0.30 \pm 0.07}$ & $(26 \pm 8) \%$ \\
Pratt+09 & $f_{\text {gas,500 }}=10^{-(1.029 \pm 0.013) h^{-1.5}(z)}\left(M_{500} /\left[2 \times 10^{14} M_{\odot}\right]\right)^{(0.21 \pm 0.03) h^{-1.5}(z)}$ & $(12 \pm 2) \%$ \\
Sun+09 (Tier 1 groups + clusters) & $f_{\text {gas,500 }}=(0.0724 \pm 0.0078) h_{73}^{-1.5}\left(M_{500} /\left[10^{13} h_{73}^{-1} M_{\odot}\right]\right)^{0.093 \pm 0.031}$ & - \\
Andreon (2010) & $f_{\text {gas }, 500}=10^{-(0.97 \pm 0.02)}\left(M_{500} /\left[10^{14.5} M_{\odot}\right]\right)^{0.15 \pm 0.03}$ & $(14 \pm 2) \%$ \\
\hline 19 clusters & $f_{*, 500}=10^{-(1.53 \pm 0.05)}\left(M_{500} /\left[10^{14} M_{\odot}\right]\right)^{-(0.49 \pm 0.09)}$ & $(20 \pm 4) \%$ \\
19 clusters-A2029-A2065 & $f_{*, 500}=10^{-(1.56 \pm 0.05)}\left(M_{500} /\left[10^{14} M_{\odot}\right]\right)^{-(0.39 \pm 0.09)}$ & $(15 \pm 29) \%$ \\
Lin+03 & $f_{*, 500}=0.0164_{-0.0090}^{+0.0010}\left(M_{500} /\left[3 \times 10^{14} M_{\odot}\right]\right)^{-(0.26 \pm 0.09)}$ & - \\
Gonzalez+07 & $f_{*, 500}=10^{7.57 \pm 0.08)} M_{500}-(0.64 \pm 0.13)$ & - \\
Giodini+09 & $f_{*, 500}=(0.050 \pm 0.001)\left(M_{500} /\left[5 \times 10^{13} M_{\odot}\right]\right)^{-(0.26 \pm 0.09)}$ & $0.35 \mathrm{dex}$ \\
Giodini+09 and Lin+03 & $f_{*, 500}=(0.050 \pm 0.001)\left(M_{500} /\left[5 \times 10^{13} M_{\odot}\right]\right)^{-(0.37 \pm 0.04)}$ & $0.50 \mathrm{dex}$ \\
Andreon (2010) & $f_{*, 500} \propto\left(M_{500} /\left[10^{14.5} M_{\odot}\right]\right)^{-(0.55 \pm 0.08)}$ & $(41 \pm 5) \%$ \\
\hline 19 clusters & $f_{\mathrm{b}, 500}=10^{-(0.73 \pm 0.24)}\left(M_{500} /\left[10^{14} M_{\odot}\right]\right)^{-(0.30 \pm 0.48)}$ & $(45 \pm 6) \%$ \\
19 clusters-A2029-A2065 & $f_{\mathrm{b}, 500}=10^{-(0.96 \pm 0.25}\left(M_{500} /\left[10^{14} M_{\odot}\right]\right)^{0.22 \pm 0.57}$ & $(29 \pm 14) \%$ \\
Lin+03 & $f_{\mathrm{b}, 500}=0.148_{-0.004}^{+0.005}\left(M_{500} /\left[3 \times 10^{14} M_{\odot}\right]\right)^{0.148 \pm 0.040}$ & - \\
Giodini+09 & $f_{\mathrm{b}, 500}=(0.123 \pm 0.003)\left(M_{500} /\left[2 \times 10^{14} M_{\odot}\right]\right)^{0.09 \pm 0.03}$ & - \\
\hline
\end{tabular}

the measured slopes of X-ray selected and near-infrared selected samples. This can either be because that the simulation sample is free of selection biases, or our sample has a sampling bias in the low-mass regime caused by low-quality statistics.

\subsection{Stellar mass fraction}

The stellar mass fraction in our observational sample decreases with increasing cluster mass; i.e., $f_{*, 500}=$ $10^{-(1.53 \pm 0.05)}\left(M_{500} /\left[10^{14} M_{\odot}\right]\right)^{-(0.49 \pm 0.09)}$ with $(20 \pm 4) \%$ intrinsic scatter for the 19 clusters. Excluding A2029 and A2065, the best fit is $f_{*, 500}=10^{-(1.56 \pm 0.05)}\left(M_{500} /\left[10^{14} M_{\odot}\right]\right)^{-(0.39 \pm 0.09)}$ with $(15 \pm 29) \%$ intrinsic scatter. We also derived the local regression fit used in Zhang et al. (2009), which demonstrates the flattening of the stellar mass fraction in the mass range lower than $2 \times$ $10{ }^{14} M_{\odot}$ (see Fig. 2). There are three situations that may introduce measurement uncertainties and affect the best fit, (1) the poor sampling in the low-mass regime, i.e., $<10^{14} M_{\odot}$; (2) the potential volume dependence in the background estimate; and (3) the two outliers for which the total masses are likely overestimated. The volume fraction of the background galaxies decreases with decreasing cluster redshift. The estimate of the local background in deriving the GLF becomes less reliable in, e.g., Coma $\left(r_{500}=41.1^{\prime}\right)$.

Within $1 \sigma$, the best fit of the stellar mass fraction as a function of the total mass agrees between our sample and the published samples (e.g., Lin et al. 2003; Gonzalez et al. 2007; Giodini et al. 2009; Andreon 2010; Dai et al. 2010) as shown in Table 3. The stellar mass fractions of nearly half of the 19 clusters in the observational sample agree with those of the simulated samples within their $1 \sigma$ interval (e.g., Fabjan et al. 2010; Puchwein et al. 2010). The amount of the stellar mass in galaxies in simulations depends rather sensitively on how the supernova feedback is included (e.g., Springel \& Hernquist 2003a; Borgani et al. 2006). For example, using kinetic feedback, e.g., in addition to the thermal feedback that Puchwein et al. (2010) used, one can obtain up to a factor of 2 fewer stars. Furthermore, the cluster mass is well defined in simulations, while there are known uncertainties in mass measurements of galaxy clusters (e.g., Biviano et al. 2006). This also causes different trends and scatter between the observational and simulated samples.

\subsection{Baryon-mass fraction}

In simulations, part of the gas does not enter the potential well of a galaxy group because gas is collisional. This phenomenon drives the so-called gas depletion. This "correction" is usually taken into account when estimating the baryon-mass fractions for observed groups. It amounts to roughly $10 \%$ within $r_{500}$ for groups (e.g., Frenk et al. 1999; Kay et al. 2004). The gas depletion is only a few percent in galaxy clusters as massive as those considered in this study (e.g., Ettori et al. 2006; Fabjan et al. 2010). We therefore do not account for it, and define the baryon-mass fraction of the galaxy cluster as the sum of the stellar mass fraction and gas-mass fraction, $f_{\mathrm{b}, 500}:=M_{*, 500} / M_{500}+$ $M_{\text {gas, } 500} / M_{500}$.

In Fig. 2, we also show the baryon-mass fraction as a function of the total mass. The baryon-mass fractions of all 19 clusters appear flat as a function of the total mass. Even when A2029 and A2065 are excluded, the best fit, $f_{\mathrm{b}, 500}=$ $10^{-(0.964 \pm 0.250)}\left(M_{500} /\left[10^{14} M_{\odot}\right]\right)^{0.224 \pm 0.567}$, still appears flat as a function of the total mass within its $1 \sigma$ error.

As shown in Table 3, the baryon-mass fractions of our sample display a similar trend as that exhibited by other observational samples (e.g., Lin et al. 2003; Gonzalez et al. 2007; Giodini et al. 2009; Andreon 2010). According to the best fit for our sample, the value reaches the WMAP 5-year measurement of the cosmic baryon-mass fraction (Dunkley et al. 2009) at $\sim 7.7 \times 10^{14} M_{\odot}$. The observational sample displays large scatter suffering from measurement uncertainties and probably also cluster physics.

\section{Discussions}

\subsection{ICL}

The small discrepancy between the baryon-mass fraction of our observational sample and the cosmic value appears to increase toward the low-mass end. The ICL is suggested to be one of the 
most important forms of missing baryons that accounts for this discrepancy, and accounts for 6-22\% of the total cluster light in the $r$-band (e.g., Krick \& Bernstein 2007; Gonzalez et al. 2007; Zibetti et al. 2005). Pierini et al. (2008) suggest that the ICL fraction may increase in cluster mergers.

Simulations with different cluster physics (e.g., Frenk et al. 1999; Borgani et al. 2004; Kay et al. 2004; Kravtsov et al. 2005; Nagai et al. 2007; Evrard et al. 2008; Sijacki et al. 2007; Fabjan et al. 2010; Kapferer et al. 2010; Puchwein et al. 2010) give different predictions. Kravtsov et al. (2005) performed numerical simulations that assume nonradiative hydrodynamics (without dissipations) in one run and radiative cooling, as well as several other physical processes in the other run. They find that the baryon-mass fraction does not depend on the cluster mass in nonradiative simulations, and that the predicted value within $r_{500}$ is $5 \%$ below the cosmic value. In simulations with radiative cooling, star formation, and AGN feedback, both the baryonmass fraction and the gas-mass fraction increase with increasing mass (e.g., Kravtsov et al. 2005; Puchwein et al. 2010). In the runs with AGN feedback, the baryon-mass fraction is close to the cosmic value for the most massive clusters, but is significantly lower than the cosmic value for low-mass systems (e.g., Fabjan et al. 2010; Puchwein et al. 2010). This is due to the amount of the ICL, as well as of a significant amount of gas removed by AGN heating from the central regions of clusters and driven to $>r_{500}$, in particular, in low-mass systems as shown by the sum of the baryon-mass fraction $\left(f_{\mathrm{gas}, 500}+f_{*, 500}\right)$ and the ICL in Fig. 5 in Puchwein et al. (2010).

In Puchwein et al. (2010), the ICL amounts to $~ 58 \%$ of the total stars, i.e., the sum of the ICL and stars in cluster galaxies, in a cluster of $4 \times 10^{13} M_{\odot}$. The amount of the stellar mass in the ICL found in simulations is enough to explain the difference between the baryon-mass fraction of our observational sample and the WMAP 5-year predicted cosmic value in the low-mass regime. However, such a large amount of the stellar mass in the ICL seems to be incompatible with recent observations (e.g., Krick \& Bernstein 2007; Gonzalez et al. 2007; Zibetti et al. 2005). This suggests that the ICL only accounts for part of the missing baryons and that other mechanisms like gas expulsion by AGN heating may be important.

\subsection{Star-formation efficiency}

We observe a decreasing stellar mass fraction as a function of the total mass (Fig. 2). The stellar mass fraction has been widely used as an estimate of the star-formation efficiency ${ }^{4}$ (e.g., Bryan 2000). There are no correlations between the stellar-to-gas mass ratio within $r_{500}$ and redshift. This indicates that the mass dependence of the star-formation efficiency is not a selection effect of redshift evolution.

Other observational samples also show varying starformation efficiency with total mass (e.g., Gonzalez et al. 2000; Lin et al. 2003; Laganá et al. 2008, 2011; Ettori et al. 2009). Dai et al. (2010) suggest that the fraction of baryon loss is determined by the depth of the potential well of the system, which leads to an increasing baryon-mass fraction as a function of the increasing total mass of a cluster. Toward the high-mass end, it approaches the cosmic value. Our finding supports two interpretations demonstrated in simulations (e.g., Fabjan et al. 2010). In massive clusters, more hot gas and dark matter is settled in the

\footnotetext{
4 The star-formation efficiency used in this work is not the stellarto-initial molecular hydrogen mass ratio, but the stellar-to-total cluster mass ratio.
}

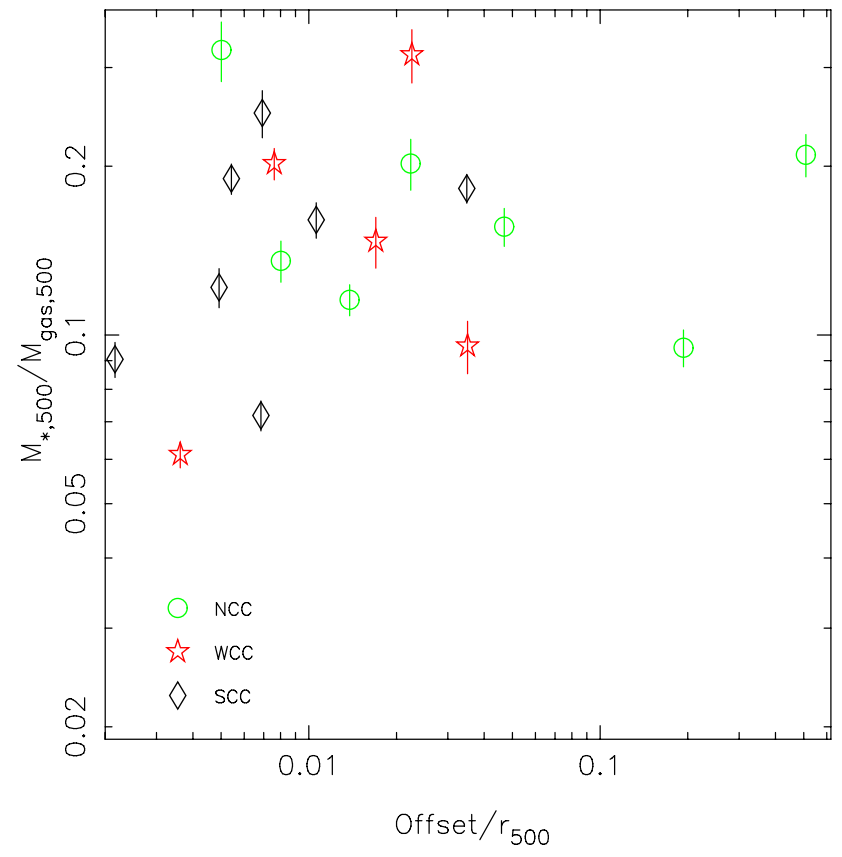

Fig. 3. Stellar-to-gas mass ratio within $r_{500}$ vs. offset between the X-ray flux-weighted center and the BCG position. The symbols have the same meaning as those in Fig. 1. The intrinsic scatter is $(41 \pm 8) \%$ and $(66 \pm 11) \%$ for the ten high- and nine low-offset systems, respectively. This indicates that the scatter of the stellar-to-gas mass ratios may reflect the freedom in the formation history of the systems since the last major merging episode.

deep potential wells than individual galaxies. In low-mass systems, the accretion of individual less massive galaxies is more important, and more low entropy gas is brought in to form stars. Both processes result in low star-formation efficiency in massive systems.

\subsubsection{Mergers and energy inputs from feedback}

In the hierarchical structure-formation scenario, the efficiency of stripping gas from member galaxies depends on the total mass of the system. In more massive clusters, galaxies exhibit higher velocity dispersion due to their deep potential wells, and as a consequence the efficiency of stripping gas from member galaxies is higher, which diminishes the star-formation efficiency in the galaxies. In low-mass systems, heating due to merging is less efficient because of their shallow potential wells. On the other hand, the effect of AGN feedback is significant in groups due to their shallow potential wells. Giodini et al. (2010) find that feedback from radio-mode AGN heating can account for the reduced gas fractions in groups in the COSMOS field. Simulations indeed show that a significant amount of gas has been removed by AGN heating from the central regions and been driven to the region beyond $r_{500}$ (e.g., Fabjan et al. 2010; Puchwein et al. 2010). Furthermore, the fraction of X-ray luminous clusters is high in the low-mass regime because of the nature of the flux selection of the HIFLUGCS. Most X-ray luminous systems are cool-core clusters and they host AGN in the central regions. There may be correlations between the stellar-to-gas mass ratio and the indicators of merging and various energy inputs from feedback.

As shown in Fig. 3, the scatter in the stellar-to-gas mass ratios appears to increase with decreasing offset between the $\mathrm{X}$-ray flux-weighted center and the bright cluster galaxy (BCG). We divided the sample into two comparable-size subsamples with a threshold of $0.01 r_{500}$ offset, and found $(41 \pm 8) \%$ and 
$(66 \pm 11) \%$ intrinsic scatter for the ten high- and nine lowoffset systems, respectively. This indicates that the scatter of the stellar-to-gas mass ratios may reflect the freedom in the formation history of the systems since the last major merging episode.

Neither the stellar-to-gas mass ratio nor the offset between the X-ray flux-weighted center and the BCG correlates with the $i$-band magnitude difference between the BCG and the second brightest cluster galaxy. There are several possible explanations. The epoch of major star formation in the progenitors of massive ellipticals may happen much earlier than they fell into the cluster potential well. There may be smoother accretion of dark matter and hot gas than individual galaxies in quiescent systems. There may be a large fraction of the stellar mass in the ICL since mergers and tidal stresses have already happened and dynamical friction had more time to operate before the time of observations.

There is no correlation between the stellar-to-gas mass ratio and the BCG magnitude. It indicates that the bulk of star formation does not dominate in the central region of the cluster, where the BCG is (also see Edwards et al. 2007; Loubser et al. 2009; Wang et al. 2010). The star-formation efficiency may be similar in the cluster core and the outskirts.

From the X-ray data we derived the cooling radius, $r_{\text {cool }}$, within which the cooling time is shorter than the age of the Universe. There is no evidence of high star-formation efficiency with increasing X-ray luminosity within the cooling radius. This indicates that radiative cooling losses within the cooling radii are offset by some heating mechanism(s).

The stellar-to-gas mass ratio decreases with increasing entropy at $0.1 r_{500}$ and $0.2 r_{500}$ (Fig. 4), i.e., $M_{*, 500} / M_{\text {gas }, 500}=$ $\left.10^{-(0.477 \pm 0.075)} S_{0.1 r_{500}} /\left[100 \mathrm{keV} \mathrm{cm}^{2}\right]\right)^{-(1.03 \pm 0.20)}$ with $(51 \pm 7) \%$ intrinsic scatter and $M_{*, 500} / M_{\text {gas }, 500}=10^{-(0.157 \pm 0.113)}\left(S_{0.2 r_{500}} /\right.$ $\left.\left[100 \mathrm{keV} \mathrm{cm}^{2}\right]\right)^{-(1.17 \pm 0.19)}$ with $(41 \pm 5) \%$ intrinsic scatter. For the cool-core clusters alone, the scatter of the stellarto-gas mass ratios becomes smaller, e.g., $M_{*, 500} / M_{\text {gas, } 500}=$ $10^{(2.18 \pm 0.49)}\left(S_{0.1 r_{500}} /\left[100 \mathrm{keV} \mathrm{cm}^{2}\right]\right)^{-(1.17 \pm 0.19)}$ with $(41 \pm 5) \%$ intrinsic scatter. The data support the interpretation that heating from merging quenches the star-formation activity of galaxies in massive systems, and feedback from supernovae and/or radio galaxies drives a significant amount of gas to the regions beyond $r_{500}$ or, alternatively, a substantially higher stellar mass fraction in the ICL is present in nonrelaxed systems (e.g., Pierini et al. 2008).

Given the evidence of AGN feedback with radio-jet powered energy input in nearby clusters (e.g., Birzan et al. 2008), we calculated the cavity power following the correlation in Birzan et al. (2008) from the radio bolometric luminosity given in Mittal et al. (2009). Unfortunately, we found no correlation between the stellar-to-gas mass ratio and the ratio between the X-ray bolometric luminosity within the cooling radius and the cavity power, respectively. The data do not support our guess on heating from the ongoing AGN feedback.

The explanation may be that the cavity power provided by recent AGN activities only affects the current star-formation rate and it has less effect on more massive systems, in which energy input from merging becomes more important. The stellarto-gas mass ratio is set by the whole star-formation and energyinput histories of the galaxy clusters, and not by the current star-formation rates of the member galaxies.

\subsubsection{Metal enrichment}

The iron abundance of the ICM cannot be measured in a homogeneous radial range for nearby clusters due to the small

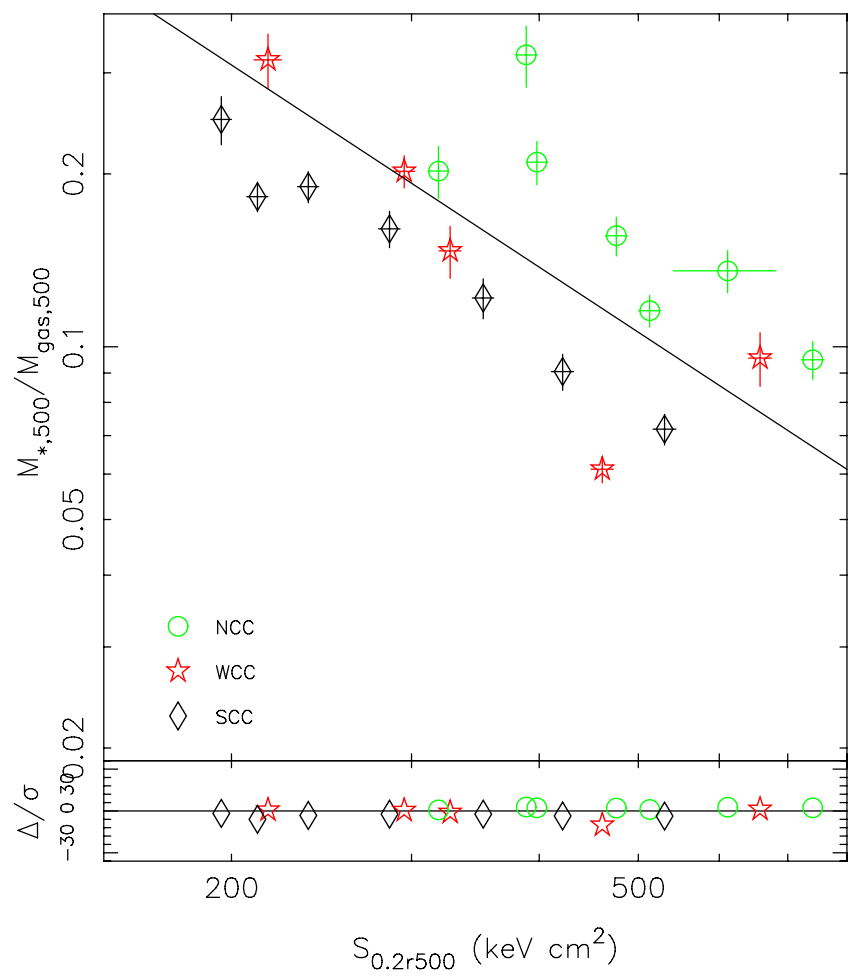

Fig. 4. Stellar-to-gas mass ratio within $r_{500}$ vs. gas entropy at $0.2 r_{500}$ and the best fit in the upper panel, as well as the residuals normalized by the error bars in the lower panel. The symbols have the same meaning as those in Fig. 1. The data support the interpretation that heating from merging quenches the star-formation activity of galaxies in massive systems, and feedback from supernovae and/or radio galaxies drives a significant amount of gas to the regions beyond $r_{500}$ or, alternatively, a substantially higher stellar mass fraction in the ICL is present in nonrelaxed systems (e.g., Pierini et al. 2008).

field-of-view (FOV) of XMM-Newton compared to the sizes of the clusters. This causes scatter in such measurements. On one hand, XMM-Newton FOV covers more of the cluster areas for lower-mass systems for a fixed redshift. Since the universal iron abundance decreases with increasing cluster radius (e.g., Kapferer et al. 2009, 2010), the measured global iron abundance within the XMM-Newton FOV is thus biased lower for low-mass systems than for high-mass systems. On the other hand, the X-ray emission of less massive systems is traced out to smaller fractions of $r_{500}$ than for more massive systems according to the flux limit. This should result in higher metallicity measurements in less massive systems when all systems can be observed out to large radii, e.g., $\sim 0.5 r_{500}$. We normalized the radius, within which the global temperature and metallicity are measured, by $r_{500}$, and found that the normalized value has no correlation with $r_{500}$. Therefore both effects are negligible for our sample.

As shown in the left hand panel in Fig. 5, we found an increasing stellar-to-gas mass ratio with decreasing cluster mass, i.e., $M_{*, 500} / M_{\text {gas }, 500}=10^{-(0.557 \pm 0.071)}\left(M_{500} /\right.$ $\left.\left[10^{14} M_{\odot}\right]\right)^{-(0.537 \pm 0.101)}$ with $(29 \pm 5) \%$ intrinsic scatter. The cool-core (classified as " $\mathrm{S}$ " in Table 1) clusters display a flatter slope, $-(0.417 \pm 0.067)$, and smaller intrinsic scatter, $<17 \%$, than the noncool-core (classified as "W" and "N" in Table 1) clusters, which show a slope of $-(0.617 \pm 0.228)$ and $(38 \pm 20) \%$ scatter. There is an increasing stellar-to-gas mass ratio with increasing iron abundance of the ICM in the right hand panel in Fig. 5; i.e., $M_{*, 500} / M_{\text {gas }, 500}=10^{-(0.106 \pm 0.174)}\left(Z / Z_{\odot}\right)^{1.53 \pm 0.38}$ with 
Y.-Y. Zhang et al.: Star-formation efficiency and metal enrichment of the ICM in local massive clusters of galaxies
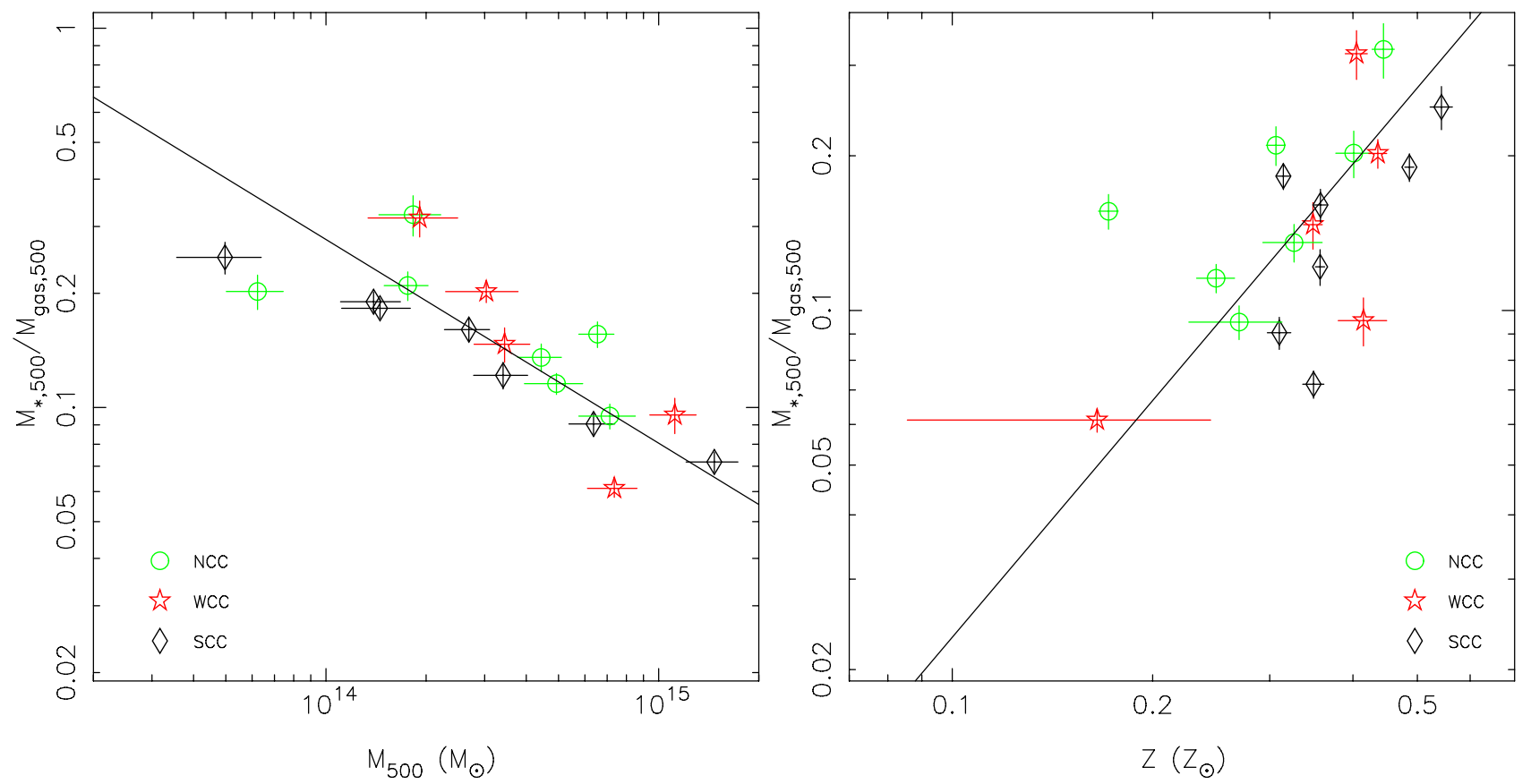

Fig. 5. Stellar-to-gas mass ratio within $r_{500}$ vs. total mass (left panel) and iron abundance (right panel) and their best fits. The symbols have the same meaning as those in Fig. 1. These two correlations indicate that the iron in the ICM mainly comes from the pollution by the star formation that happened in the past. In less massive galaxy systems, the star formation efficiency is higher.
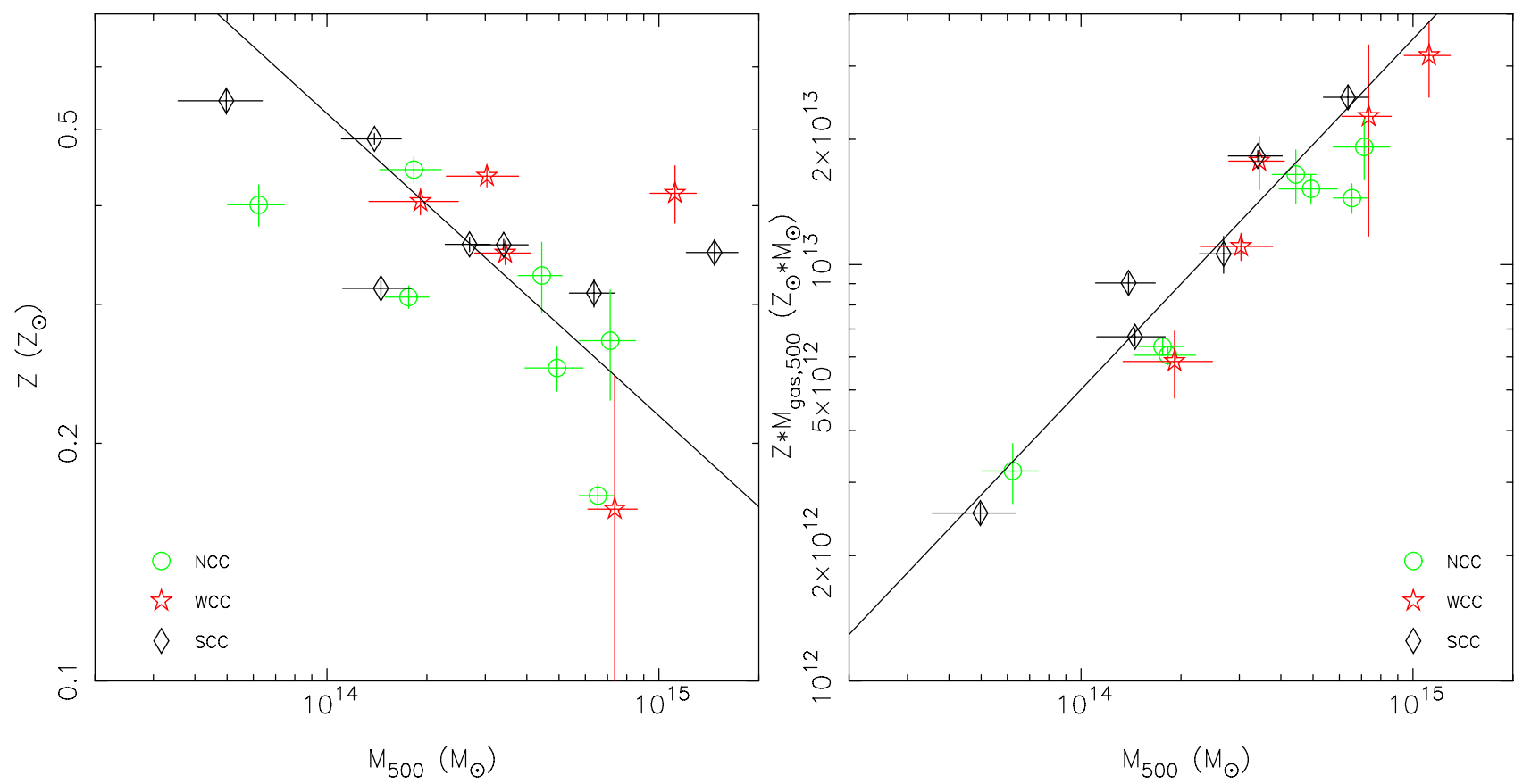

Fig. 6. Iron abundance (left panel) and iron mass (right panel) vs. total mass and their best fits. The symbols have the same meaning as those in Fig. 1. This finding agrees with the trend seen in simulations (e.g., Fig. 11 in Fabjan et al. 2010) toward less massive clusters having lower gas-mass fractions but higher iron-mass fractions, so that the gas is more metal-rich in those systems.

$(44 \pm 6) \%$ intrinsic scatter. We note that the trend holds even when one looks at the cool-core clusters and noncool-core clusters, respectively. It is interesting that the scatter of the cool-core clusters and noncool-core clusters is comparable.

The above two correlations indicate that the iron in the ICM mainly comes from the pollution by the star formation that happened in the past. In less massive galaxy systems, the star formation efficiency is higher. In other words, more stars were formed that have delivered more metals to enrich the hot gas.
In high-mass clusters, energy feedback from, e.g., merging, may quench star formation in their member galaxies, which results in low star-formation efficiency and less metal enrichment in the hot gas by stars. In addition, a larger amount of hot gas is accreted in a more massive system due to its deeper potential well, which dilutes the iron abundance more.

As shown in Fig. 6, the observational sample shows decreasing iron abundance with increasing total cluster mass, i.e., $Z / Z_{\odot}=10^{-(0.28 \pm 0.07)}\left(M_{500} /\left[10^{14} M_{\odot}\right]\right)^{-(0.383 \pm 0.101)}$ with 


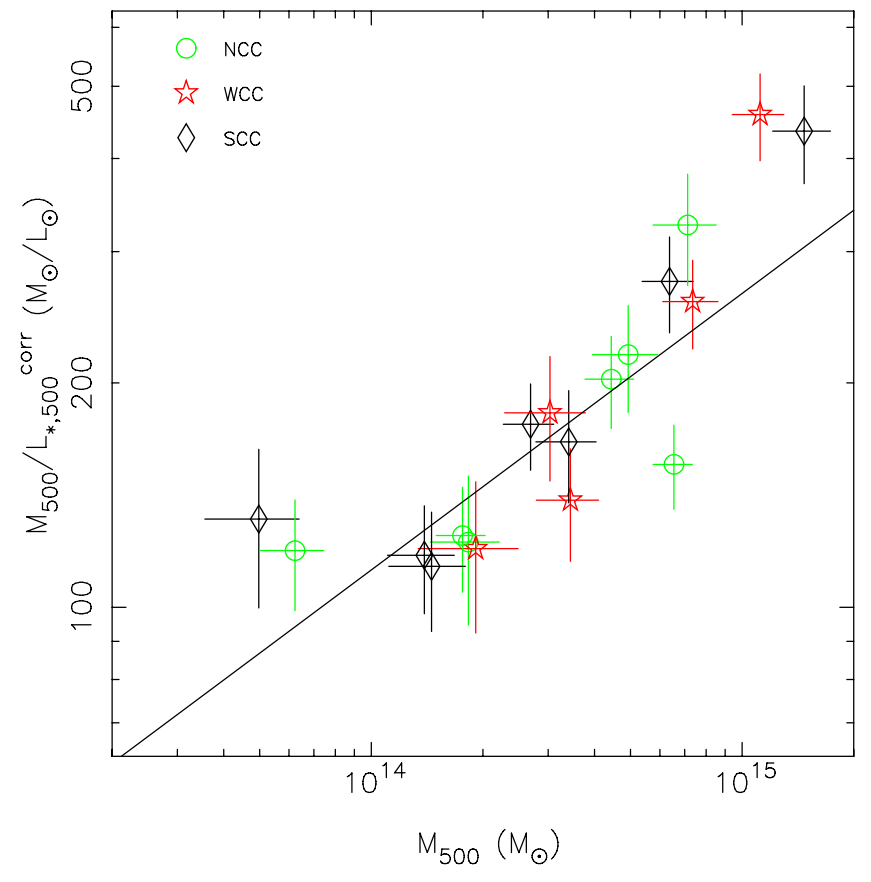

Fig. 7. Total mass-to-optical light ratio within $r_{500}$ vs. total mass and the best fit excluding A2029 and A2065. The symbols have the same meaning as those in Fig. 1. The behavior of the total mass-to-optical light ratio supports the variation in star-formation efficiencies.

$(38 \pm 5) \%$ intrinsic scatter, and increasing iron mass with increasing total cluster mass, i.e., $Z M_{\text {gas }, 500} /\left[Z_{\odot} M_{\odot}\right]=$ $10^{12.7 \pm 0.03}\left(M_{500} /\left[10^{14} M_{\odot}\right]\right)^{0.842 \pm 0.049}$ with $(17 \pm 3) \%$ intrinsic scatter. This finding agrees with the trend seen in simulations (e.g., Fig. 11 in Fabjan et al. 2010) that less massive clusters have lower gas-mass fractions but higher iron-mass fractions, so that the gas is more metal-rich in those systems. This can be explained in two ways. One is that the star-formation efficiency is high in low-mass systems: more gas is converted into stars within $r_{500}$ which yields more metals that enrich the gas. Alternatively, metal-poor hot gas has not fallen into the potential wells in low-mass systems. Most likely, metal-loaded outflows from galaxies started well before the potential well of a cluster was settled (see Noll et al. 2009), so that the metal enrichment of the observed ICM reflects a heavier star-formation activity in low-mass systems. Accordingly, the total mass-to-optical light ratio decreases with decreasing cluster mass (Fig. 7).

\subsubsection{Total mass-to-optical light ratio}

Optical and near-infrared observations suggest that the total mass-to-optical light ratio, i.e., $M / L_{*}^{\text {corr }}$, increases with cluster mass with a slope in the range of $0.2-0.4$ assuming a power-law relation (e.g., Adami et al. 2009; Bahcall \& Comerford 2002; Rines et al. 2004). In Fig. 7, we show the total mass-to-optical light ratio as a function of the total mass for our sample, which follows a trend of $M_{500} / L_{*, 500}^{\text {corr }}=$ $10^{2.02 \pm 0.05}\left(M_{500} /\left[10^{14} M_{\odot}\right]\right)^{0.468 \pm 0.085}$ with $(17 \pm 4) \%$ intrinsic scatter. When A2029 and A2065 are excluded, the best fit is $M_{500} / L_{*, 500}^{\text {corr }}=10^{2.05 \pm 0.05}\left(M_{500} /\left[10^{14} M_{\odot}\right]\right)^{0.371 \pm 0.098}$ with $(12 \pm 10) \%$ intrinsic scatter.

The behavior of the total mass-to-optical light ratio supports the variation in star-formation efficiencies found in simulations (e.g., Springel \& Hernquist 2003a; Saro et al. 2006). More massive clusters host more massive galaxies dominated by old, passively evolving stellar populations, where optical light has declined more significantly than the average coeval galaxy due to their earlier formation time. Alternatively, more massive clusters may have accreted many more low-mass galaxies, or inhibited star formation in intermediate-mass/low-mass galaxies in a more efficient way, which leads to higher total mass-to-optical light ratios in massive systems.

The scatter in the total mass-to-optical light ratio vs. total mass may indicate the coupling between the stochastic nature of the assembly times of dark matter halo and stellar mass. The best fit excluding A2029 and A2065 leads to $M_{500} / L_{*, 500}^{\text {corr }} \sim 264$ for a system of $10^{15} M_{\odot}$. Given the galaxy luminosity density and the assumption of an equivalent total mass-to-optical light ratio of the Universe to our extrapolated value for a $M_{500}=10^{15} M_{\odot}$ system, this results in a consistent $\Omega_{\mathrm{m}}$ with the concordance value within the scatter (also see e.g., Girardi et al. 2000; Reiprich 2003).

\section{Conclusions}

We have investigated the baryon content of a sample of 19 clusters selected from an X-ray flux-limited sample of 64 nearby clusters, in which we measured the cluster total masses and radii based on the optical spectroscopic data, which are independent of both the X-ray XMM-Newton and ROSAT data that we used to derive X-ray quantities, such as the gas masses, and of the SDSS DR7 imaging data that we used to compute the stellar masses. We summarize our results and interpretations as follows.

- The gas-mass fraction of the observational sample increases with increasing cluster mass. The observed gas-mass fractions of our sample agree with previous observational results (e.g., Sun et al. 2007; Pratt et al. 2009). The values of our observational sample are also in good agreement with those of the simulated samples (e.g., Fabjan et al. 2010; Puchwein et al. 2010) in the high-mass regime.

- The stellar mass fraction decreases with increasing cluster mass and exhibits large scatter $(20 \pm 4 \%)$. The observed stellar mass fractions of our sample agree with previous observational results and the results of the simulated clusters (e.g., Fabjan et al. 2010; Puchwein et al. 2010) within the scatter.

- We observe lower stellar-to-gas mass ratios in those systems that display higher central entropies indicating energy inputs from feedback. This supports a twofold interpretation: heating from merging quenches the star formation in galaxies in massive systems, and feedback from supernovae and/or radio galaxies removes a significant amount of gas in lowmass systems or, alternatively, a substantially higher stellar mass fraction in the ICL is associated with dynamically active systems of galaxies.

- Less massive clusters are confirmed to host less gas per unit total mass; however, they exhibit higher metal-mass fractions, so that their ICM is more metal-rich. Member galaxies of low-mass systems may be forming stars more efficiently, thereby producing more metals, part of which are ejected from the galaxies and enriched the ICM to a higher level. Alternatively, some gas could have been expelled from these systems or be missing from the hot phase. The former hypothesis is preferred as the main driver of the massdependent metal enrichment since the total mass-to-optical luminosity ratio increases as the total mass increases.

Acknowledgements. The XMM-Newton project is an ESA Science Mission with instruments and contributions directly funded by ESA Member States and the 
USA (NASA). The XMM-Newton project is supported by the Bundesministerium für Wirtschaft und Technologie/Deutsches Zentrum für Luft- und Raumfahrt (BMWI/DLR, FKZ 50 OX 0001) and the Max-Planck Society. Part of the simulations, to which our results were compared, were performed by Debora Sijacki on the Cambridge high-performance computing cluster Darwin. Y.Y.Z. acknowledges Pavel Kroupa and Stefano Borgani for constructive discussions and Jacopo Fritz for providing the best-fit relation between stellar masses computed from the SDSS DR7 photometric data and spectroscopic data. Y.Y.Z. acknowledges support from the German BMBF through the Verbundforschung under grant No. 50 OR 1005 and travel support from the Deutsche Forschungsgemeinschaft Priority Program 1177 (Witnesses of Cosmic History: Formation and Evolution of Galaxies, Black Holes and Their Environment). T.F.L acknowledges support from the FAPESP through grants 2006/56213-9 and 2008/04318-7, as well as from the CAPES through BEX3405-10-9. D.P. acknowledges support from the German BMBF through the Verbundforschung under grant No. 50 OR 0405 and the kind hospitality of the MPE. E.P. is supported by the DFG through Transregio 33. T.H.R. acknowledges support by the DFG through Heisenberg grant RE 1462/5.

\section{References}

Abazajian, K. N., Adelman-McCarthy, J. K., Agüeros, M. A., et al. 2009, ApJS, 182,543

Adami, C., Biviano, A., \& Mazure, A. 1998, A\&A, 331, 439

Akritas, M. G., \& Bershady, M. A. 1996, ApJ, 470, 706

Andreon, S. 2010, MNRAS, 407, 263

Arnaboldi, M., \& Gerhard, O. 2010, Proc. International Astronomical Union 2009, 5, 97

Arnaud, M., Pointecouteau, E., \& Pratt, G. W. 2007, A\&A, 474, L37

Bahcall, N. A. 1977, ApJ, 218, L93

Bahcall, N. A., \& Comerford, J. M. 2002, ApJ, 565, L5

Bell, E. F., \& de Jong, R. S. 2001, ApJ, 550, 212

Bell, E. F., McIntosh, D. H., Katz, N., \& Weinberg, M. D. 2003, ApJS, 149, 289

Bertelli, G., Bressan, A., Chiosi, C., Fagotto, F., \& Nasi, E. 1994, A\&AS, 106, 275

Birzan, L., McNamara, B. R., Nulsen, P. E. J., Carilli, C. L., \& Wise, M. W. 2008, ApJ, 686, 859

Biviano, A., Murante, G., Borgani, S., et al. 2006, A\&A, 456, 23

Blanton, M. R., Dalcanton, J., Eisenstein, D., et al. 2001, AJ, 121, 2358

Blanton, M. R., Hogg, D. W., Bahcall, N. A., et al. 2003, ApJ, 592, 819

Böhringer, H., Schuecker, P., Pratt, G. W., et al. 2007, A\&A, 469, 363

Borgani, S., Murante, G., Springel, V., et al. 2004, MNRAS, 348, 1078

Borgani, S., Dolag, K., Murante, G., et al. 2006, MNRAS, 367, 1641

Bruzual, G., \& Charlot, S. 2003, MNRAS, 344, 1000

Bryan, G. L. 2000, ApJ, 544, L1

Cappellari, M., Bacon, R., Bureau, M., et al. 2006, MNRAS, 366, 1126

Cole, S., Norberg, P., Baugh, C. M., et al. 2001, MNRAS, 326, 255

Dahle, H. 2006, ApJ, 653, 954

Dai, X., Bregman, J. N., Kochanek, C. S., \& Rasia, E. 2010, ApJ, 719, 119

David, L. P., Arnaud, K. A., Forman, W., \& Jones, C. 1990, ApJ, 356, 32

De Filippis, E., Paolillo, M., Longo, G., et al. 2011, MNRAS, 414, 2771

Dietrich, J. P., Biviano, A., Popesso, P., et al. 2009, A\&A, 499, 669

Dolag, K., Bartelmann, M., Perrotta, F., et al. 2004, A\&A, 416, 853

Dolag, K., Murante, G., \& Borgani, S. 2010, MNRAS, 405, 1544

Dressler, A. 1980, ApJ, 236, 351

Dressler, A., \& Shectman, S. A. 1988, AJ, 95, 985

Dunkley, J., Komatsu, E., Nolta, M. R., et al. 2009, ApJS, 180, 306

Edwards, L. O. V., Hudson, M. J., Balogh, M. L., \& Smith, R. J. 2007, MNRAS, 379,100

Ettori, S., De Grandi, S., \& Molendi, S. 2002, A\&A, 391, 841

Ettori, S., Dolag, K., Borgani, S., \& Murante, G. 2006, MNRAS, 365, 1021

Ettori, S., Morandi, A., Tozzi, P., et al. 2009, A\&A, 501, 61

Evrard, A. E., Bialek, J., Busha, M., et al. 2008, ApJ, 672, 122

Fabjan, D., Borgani, S., Tornatore, L., et al. 2010, MNRAS, 401, 1670

Finoguenov, A., Reiprich, T. H., \& Böhringer, H. 2001, A\&A, 368, 749

Frenk, C. S., White, S. D. M., Bode, P., et al. 1999, ApJ, 525, 554

Fritz, J., Poggianti, B. M., Cava, A., et al. 2011, A\&A, 526, A45

Gastaldello, F., Buote, D. A., Humphrey, P. J., et al. 2007, ApJ, 669, 158

Gerhard, O., Kronawitter, A., Saglia, R. P., \& Bender, R. 2001, AJ, 121, 1936

Giodini, S., Pierini, D., Finoguenov, A., \& Pratt, G. W. 2009, ApJ, 703, 982
Giodini, S., Smolčić, V., Finoguenov, A, et al. 2010, ApJ, 714, 218

Girardi, M., Giuricin, G., Mardirossian, F., Mezzetti, M., \& Boschin, W. 1998, ApJ, 505, 74

Girardi, M., Borgani, S., Giuricin, G., Mardirossian, F., \& Mezzetti, M. 2000, ApJ, 530, 62

Gonzalez, A. H., Zabludoff, A. I., Zaritsky, D., \& Dalcanton, J. J. 2000, ApJ 536,561

Gonzalez, A. H., Zaritsky, D., \& Zabludoff, A. I. 2007, ApJ, 666, 147

Hudson, D. S., Mittal, R., Reiprich, T. H., et al. 2010, A\&A, 513, 37

Kapferer, W., Kronberger, T., Breitschwerdt, D., et al. 2009, A\&A, 504, 719

Kapferer, W., Schindler, S., Knollmann, S. R., et al. 2010, A\&A, 516, A41

Kauffmann, G., Heckman, T. M., White, S. D. M., et al. 2003, MNRAS, 341, 33

Kay, S. T., Thomas, P. A., Jenkins, A., \& Pearce, F. R. 2004, MNRAS, 355, 1091

Kennicutt, R. C. 1983, ApJ, 272, 54

Kochanek, C. S., Pahre, M. A., Falco, E. E., et al. 2001, ApJ, 560, 566

Kravtsov, A. V., Nagai, D., \& Vikhlinin, A. 2005, ApJ, 625, 588

Krick, J. E., \& Bernstein, R. A. 2007, AJ, 134, 466

Kroupa, P. 2001, MNRAS, 322, 231

Laganá, T. F., Lima Neto, G. B., Andrade-Santos, F., \& Cypriano, E. S. 2008, A\&A, 485, 633

Laganá, T. F., Zhang, Y.-Y., Reiprich, T. H., \& Scheider, P. 2011, ApJ, accepted [arXiv: 1108.3678$]$

Lin, Y.-T., Mohr, J. J., \& Stanford, S. A. 2003, ApJ, 591, 749

Loubser, S. I., Sánchez-Blázquez, P., Sansom, A. E., \& Soechting, I. K. 2009, MNRAS, 398, 133

Macciò, A. V., Dutton, A. A., \& van den Bosch, F. C. 2008, MNRAS, 391, 1940

Mahdavi, A., Hoekstra, H., Babul, A., \& Henry, J. P. 2008, MNRAS, 384, 1567

Mittal, R., Hudson, D. S., Reiprich, T. H., \& Clarke, T. 2009, A\&A, 501, 835

Murante, G., Arnaboldi, M., Gerhard, O., et al. 2004, ApJ, 607, L83

Nagai, D., Vikhlinin, A., \& Kravtsov, A. V. 2007, ApJ, 655, 98

Navarro, J. F., Frenk, C. S., \& White, S. D. M. 1997, ApJ, 490, 493

Neto, A. F., Gao, L., Bett, P., et al. 2007, MNRAS, 381, 1450

Noll, S., Pierini, D., Cimatti, A., et al. 2009, A\&A, 499, 69

Oemler, A. J. 1974, ApJ, 194, 1

Paolillo, M., Andreon, S., Longo, G., et al. 2001, A\&A, 367, 59

Pierini, D., Zibetti, S., Braglia, F., et al. 2008, A\&A, 483, 727

Poggianti, B. M. 1997, A\&AS, 122, 399

Popesso, P., Biviano, A., Böhringer, H., Romaniello, M., \& Voges, W. 2005, A\&A, 433, 431

Pratt, G. W., Croston, J. H., Arnaud, M., \& Böhringer, H. 2009, A\&A, 498, 361

Puchwein, E., Sijacki, D., \& Springel, V. 2008, ApJ, 687, L53

Puchwein, E., Springel, V., Sijacki, D., \& Dolag, K. 2010, MNRAS, 406, 936

Reiprich, T. H. 2003, Ph.D. Thesis [arXiv: astro-ph/0308137]

Reiprich, T. H., \& Böhringer, H. 2002, ApJ, 567, 716

Rines, K., \& Diaferio, A. 2006, AJ, 132, 1275

Rines, K., Geller, M. J., Diaferio, A., Kurtz, M. J., \& Jarrett, T. H. 2004, AJ, 128, 1078

Roussel, H., Sadat, R., \& Blanchard, A. 2000, A\&A, 361, 429

Salpeter, E. E. 1955, ApJ, 121, 161

Sarazin, C. L., \& Bahcall, J. N. 1977, ApJS, 34, 451

Saro, A., Borgani, S., Tornatore, L., et al. 2006, MNRAS, 373, 397

Schechter, P. 1976, ApJ, 203, 297

Sijacki, D., Springel, V., Di Matteo, T., \& Hernquist, L. 2007, MNRAS, 380, 877

Springel, V., \& Hernquist, L. 2003a, MNRAS, 339, 289

Springel, V., \& Hernquist, L. 2003b, MNRAS, 339, 312

Sun, M., Donahue, M., Voit, G. M., et al. 2007, ApJ, 671, 190

Sun, M., Voit, G. M., Donahue, M., et al. 2009, ApJ, 693, 1142

Vikhlinin, A., Kravtsov, A., Forman, W., et al. 2006, ApJ, 640, 691

Vikhlinin, A., Burenin, R. A., Ebeling, H., et al. 2009, ApJ, 692, 1033

Wang, J., Overzier, R., Kauffmann, G., von der Linden, A., \& Kong, X. 2010, MNRAS, 401, 433

Wu, X.-P., Xue, Y.-J., \& Fang, L.-Z. 1999, ApJ, 524, 22

Zhang, Y.-Y., Böhringer, H., Finoguenov, A., et al. 2006, A\&A, 456, 55

Zhang, Y.-Y., Finoguenov, A., Böhringer, H., et al. 2008, A\&A, 482, 451

Zhang, Y.-Y., Reiprich, T. H., Finoguenov, A., Hudson, D. S., \& Sarazin, C. L. 2009, ApJ, 699, 1178

Zhang, Y.-Y., Okabe, N., Finoguenov, A., et al. 2010, ApJ, 711, 1033

Zhang, Y.-Y., Andernach, H., Caretta, C. A., et al. 2011, A\&A, 526, A105

Zibetti, S., White, S. D. M., Schneider, D. P., \& Brinkmann, J. 2005, MNRAS, 358, 949

Zwicky, F. 1957, Morphological Astronomy (Berlin: Springer) 